\section{A brighter future for the implementation of pharmacogenomic testing}

\author{
Cathelijne H van der Wouden ${ }^{1}$, Jesse J Swen ${ }^{1}$, Matthias Samwald ${ }^{2}$, \\ Christina Mitropoulou ${ }^{3}$, Matthias Schwab ${ }^{4,5}$ and Henk-Jan Guchelaar ${ }^{1}$ \\ on behalf of the Ubiquitous-Pharmacogenomics Consortium
}

European Journal of Human Genetics (2016) 24, 1658-1660; doi:10.1038/ejhg.2016.116; published online 31 August 2016

$\mathrm{T}$ he study reported by Kapoor et al in this month's European Journal of Human Genetics explains the dichotomy between the accelerated pace of discovery and the slow uptake of pharmacogenomics in standard clinical care through a SWOT analysis. A glass can be perceived as being half-full or half-empty. Although the authors predict many positive effects and applications of pharmacogenomics they also present a rather pessimistic view on the status and attitudes toward pharmacogenomics and its implementation in clinical care. However, we, like many others, foresee a much more optimistic future for both the impact of pharmacogenomics and its imminent implementation. ${ }^{2}$ Our optimism is based on the fact that many of the proposed weaknesses and threats are currently being addressed by various initiatives, both in the United States of America and in Europe, and that the presented hurdles preventing implementation such as lack of evidence regarding clinical utility, absence of guidelines within a clinical workflow and the necessity of whole-genome sequencing (WGS) innovations in pharmacogenomics are either being overcome or have already been surmounted.

Indeed, the optimistic prediction for the imminent implementation and resulting impact of pharmacogenomics on health care is shared by many stakeholders. This is verified by the recent funding of eight implementation initiatives in the United States of America over the last five years; such as the PREDICT program, highlighted by Kapoor et al. ${ }^{1}$ The US Pharmacogenomics Research Network (PGRN), has initiated the Translational Pharmacogenetics Program aimed at implementing pre-emptive pharmacogenomic testing in several clinical US sites in a real-world setting. ${ }^{3}$ In addition, the 1200 Patients Project initiated by the University of Chicago is implementing pre-emptive pharmacogenomic testing of a panel of pharmacogenes. ${ }^{4,5}$ The Displaying and Integrating Genetic Information Through the Electronic Health Record (EHR) Action Collaborative (DIGITiZe) focuses on integrating and $H L A-B^{\star} 5701$ into the EHR for patients receiving prescriptions for azathioprine and abacavir. ${ }^{6}$ The Electronic Medical Records and Genomics PGx project (eMERGE-PGx), initiated in ten US medical centres, will apply next-generation sequencing (NGS) for 84 proposed pharmacogenes in 9000 patients likely to be prescribed relevant drugs in a one- to three-year time frame. ${ }^{7}$ The PG4KDS project at St Jude Children's Hospital aims at implementing pharmacogenomic testing for genes corresponding to 12 high-risk drugs. ${ }^{8}$ pharmacogenomic testing results of TPMT

${ }^{1}$ Department of Clinical Pharmacy \& Toxicology, Leiden University Medical Center, Leiden, The Netherlands; ${ }^{2}$ Center for Medical Statistics, Informatics, and Intelligent Systems, Medical University of Vienna, Vienna, Austria; ${ }^{3}$ The Golden Helix Foundation, London, UK; ${ }^{4}$ Dr Margarete Fischer-Bosch Institute of Clinical Pharmacology, Stuttgart, Germany; ${ }^{5}$ Department of Clinical Pharmacology, University Hospital Tuebingen, Tuebingen, Germany

*Correspondence: Professor Dr H-J Guchelaar, Department of Clinical Pharmacy \& Toxicology, Leiden University Medical Center, PO Box 9600, NL 2300 RC Leiden, The Netherlands. Tel: +31 (0)71 526 2790; Fax: +31 (0)71 526 6980; E-mail: H.J.Guchelaar@lumc.nl

Received 30 June 2016; revised 5 July 2016; accepted 22 July 2016; published online 31 August 2016
The Clinical Implementation of Personalized Medicine through Electronic Health Records and Genomics - Pharmacogenomics (CLIPMERGE) project at Mount Sinai Medical Center aims at enrolling patients who have previously opted-in to the institution's biobank and to implement pharmacogenomic testing within the hospital. ${ }^{9}$ The Personalized Medicine Program at the University of Florida and Shands Hospital has implemented CYP2C19 genotyping for patients who have been prescribed clopidogrel, as a pilot study. This project aims to expand on the implementation to a variety of other practice settings. ${ }^{10,11}$ The PREDICT program established at the Vanderbilt University School of Medicine aims to implement pre-emptive genotyping for high-impact genetic variants. The project initially focused on CYP2C19 genotyping of patients scheduled for coronary arteriography. ${ }^{12}$ All these initiatives have three similar approaches in common, as follows: (1) they implemented pre-emptive pharmacogenomic testing using either NGS, a panel of pharmacogenes or a single drug-gene combination in clinical care, (2) they embedded the pharmacogenomics results within the EHR and combined it with clinical decision support systems (CDSS) and (3) they used Clinical Pharmacogenomics Implementation Consortium (CPIC) guidelines to guide drug selection and dosing.

Now, the first large-scale, international pharmacogenomics implementation project has been initiated in Europe. The European Commission recently awarded a 15 million Euro Horizon 2020 grant to fund the Ubiquitous Pharmacogenomics Consortium (U-PGx) 'Making actionable pharmacogenomics data and effective treatment optimization accessible to every European citizen' (www.upgx.eu). In contrast to the US initiatives, the U-PGx consortium will not only implement pharmacogenomics tests for a broad range of drugs but also accept the challenge of assessing the impact of pharmacogenomics on health outcomes using a novel composite endpoint. The U-PGx project uses a multifaceted approach, consisting of four components, to address the major challenges and obstacles for implementation of pharmacogenomics in patient care. The first component focuses on developing the enabling tools necessary to integrate pharmacogenomic test results into the EHR and the CDSS such as IT solutions and educating health-care professionals in pharmacogenomics, as well as complementary mobile solutions where centralized EHRs are not available. The second component is a randomized controlled trial ( $n=8000$ ), which will assess the impact of 
pre-emptive pharmacogenomic testing of a panel of 13 pharmacogenes and the corresponding drug-gene guidelines for 45 drugs, originating from the Dutch Pharmacogenomics Working Group (DPGW) on patient outcomes in seven European countries. ${ }^{13,14}$ In addition, a health technology assessment will be performed and process indicators for physician adoption of pharmacogenomics will be monitored. The results of this approach will provide regulatory authorities with evidence on both the patient and economic impact of pharmacogenomics to enable evidence-based decision-making to shape policy. A third component applies innovative methodologies, such as systems pharmacology and NGS, to discover additional variants associated with drug response and to elucidate drug-drug-gene interactions. The last component assures ethical proceeding of the project and spearheads outreaching and educational activities to influential stakeholders. The funding of this large consortium demonstrates both the expectations of the impact of pharmacogenomics and the importance for many stakeholders. In a time where increasing health-care costs are becoming a significant problem, pharmacogenomics can be used to personalize treatment modalities and thereby minimize adverse drug reactions, resulting in increased quality of life and lowered health-care expenditure.

Kapoor et $a l^{1}$ describe the demonstration of clinical utility as a necessary step in establishing a pharmacogenomic indication. In our opinion, the association between a pharmacogenotype and drug response does not ultimately require the merit of a large randomized clinical trial to highlight its utility. This view is shared by others. ${ }^{15,16}$ For example, the clinical utility of using renal function to guide drug dosage, in avoiding toxicity, has not been evaluated though large randomized controlled trials as a regulatory requirement but rests purely upon pharmacological knowledge of health-care professionals. Similarly, we suggest that using pharmacogenomic and pharmacological knowledge is sufficient to evaluate the clinical utility of modifying dosage on the basis of a pharmacogenomic genotype result. The evidence that the pharmacogenomics approach does indeed result in a positive benefit-risk ratio is already given by the randomized controlled trials evaluating the association between CYP2D6, VKORC1, TPMT, HLA$B^{\star} 5701$ and the respective drug response. ${ }^{17-20}$ We hypothesize that this gold-standard evidence for pharmacogenomics-guided prescribing can be extrapolated to all single pharmacogenomic interventions. This hypo- thesis will be put to the test in the U-PGX clinical trial where the combined effect of multiple pharmacogenomic interventions will be assessed aggregately. In addition, the use of WGS in oncology and whole-exome sequencing in clinical genetics often results in complementary pharmacogenomics information being provided. ${ }^{21}$ Once these pharmacogenomic data are already available, the discussion on clinical utility and costeffectiveness are superfluous. It seems, however, that health-care professionals are more uncomfortable with applying genetics than applying other tools, such as renal function, due to perceived complexity surrounding the domain of genetics. We, therefore, argue that the bottleneck for the implementation of pharmacogenomics does not lie in the lack of evidence demonstrating clinical utility but rather lies in the unfamiliarity and lack of genetics knowledge among physicians and other health-care providers. ${ }^{22-24}$ This bottleneck will be addressed within the U-PGX consortium through an e-learning tool, which aims to increase physician knowledge and know-how with pharmacogenomics.

The lack of guidelines within a clinical workflow is a hurdle that needs to be overcome to enable successful implementation of pharmacogenomics and to guide health-care professionals in using pharmacogenomics in clinical care, as described by Kapoor et al. ${ }^{1}$ The CPIC has developed 325 drug-gene guidelines, which can be accessed through the PharmGKB. Simultaneously, the DPWG has developed over 80 drug-gene guidelines in The Netherlands. ${ }^{13,14}$ These are integrated nationwide within the Dutch Z-index, the national drug database used for electronic prescribing by physicians and medication surveillance by pharmacists, and are, therefore, incorporated in the CDSS. This gives Dutch health-care providers the opportunity to easily incorporate pharmacogenotypes in their prescribing and dispensing. This, however, has still not resulted in the adoption of these guidelines in clinical care. Some major obstacles of implementation can be identified from the following: (1) a lack of knowledge among health-care providers, as mentioned earlier and (2) the absence of the patient's genotype at the time of prescribing or dispensing. We, therefore, argue that pre-emptive genotyping is more effective than reactive genotyping with regard to the implementation of pharmacogenomics. A recent study shows that over $97 \%$ of the population carries an actionable pharmacogenomic variant. ${ }^{25}$ This implies that large portions of the population will benefit from pre-emptive genotyping at some point in their lifetime. The U-PGx consortium will develop a range of tools to be able to implement these guidelines and combine them with the pre-emptively acquired genotype in a range of different medical systems across seven European countries. These tools vary from being interruptive within the CDSS to a tool which is carried by the patient on an ID card and can be accessed through a QR code.

Obviously, pharmacogenomics is still a novel discipline and will be further developed over the next years. The incomplete understanding of the genetic impact on drug responses limits the benefits of pharmacogenomics in clinical care. Through discovering more particularly rare variants associated with drug response and using systems pharmacology approaches, we are able to increase the understanding of pharmacogenomics and, thereby, increase its benefits and impact. The U-PGx consortium will achieve this by using two approaches: (1) by using WGS to identify rare variants that are associated with drug response and (2) by using systems pharmacology models including multiple variables such as co-medication, gender and age to discover and elucidate drug-drug-gene interactions. The use of WGS may at present not be a cost-effective approach with regard to pharmacogenomic-guided prescribing and dispensing. However, as time passes the cost of this technology will asymptotically approach a point where the application of WGS for the use of pharmacogenomics will not be cost driven but rather technology driven.

Kapoor et $a l^{1}$ conclude their analysis with recommending an evidenced-based approach that interrogates whether the pharmacogenomic test genuinely improves quality of life and health care in a cost-effective manner to convince regulation authorities on implementation. As mentioned earlier, we argue that providing evidence of clinical utility for a single pharmacogenomic test is unnecessary and excessive. We, however, do agree that evidence should be provided for regulation authorities. The U-PGx trial is, therefore, primarily designed to quantify the aggregate effect of multiple pre-emptive pharmacogenomic tests on health outcome. Secondarily, it will evaluate the cost-effectiveness of this pharmacogenomic intervention. Through U-PGx we are confident to make physicians more comfortable with pharmacogenomic testing and its implementation in standard care and consequently make pharmacogenomic testing accessible to all European citizens. 


\section{CONFLICT OF INTEREST}

Matthias Schwab is the contributor to patents related to TPMT. The remaining authors declare no conflict of interest.

\section{ACKNOWLEDGEMENTS}

The research leading to these results has received funding from the European Community's Horizon 2020 Programme under grant agreement No. 668353 (U-PGx).

\section{Kapoor et al: EJHG.}

2 Kampourakis K, Vayena E, Mitropoulou C et al: Key challenges for next-generation pharmacogenomics: science \& society series on science and drugs. EMBO Rep 2014; 15: 472-476.

3 Shuldiner AR, Relling MV, Peterson JF et al: The Pharmacogenomics Research Network Translational Pharmacogenetics Program: overcoming challenges of real-world implementation. Clin Pharmacol Ther 2013; 94: 207-210.

4 O'Donnell PH, Bush A, Spitz J et al: The 1200 patients project: creating a new medical model system for clinical implementation of pharmacogenomics. Clin Pharmacol Ther 2012; 92: 446-449.

5 O'Donnell PH, Danahey K, Jacobs M et al: Adoption of a clinical pharmacogenomics implementation program during outpatient care-initial results of the University of Chicago "1,200 Patients Project". Am J Med Genet C Semin Med Genet 2014; 166C: 68-75.

6 Bell GC, Crews KR, Wilkinson MR et al: Development and use of active clinical decision support for preemptive pharmacogenomics. J Am Med Inform Assoc 2014; 21: e93-e99.
7 Rasmussen-Torvik LJ, Stallings SC, Gordon AS et al: Design and anticipated outcomes of the eMERGE-PGx project: a multicenter pilot for preemptive pharmacogenomics in electronic health record systems. Clin Pharmacol Ther 2014; 96: 482-489.

8 Hoffman JM, Haidar CE, Wilkinson MR et al: PG4KDS: a model for the clinical implementation of pre-emptive pharmacogenetics. Am J Med Genet C Semin Med Genet 2014; 166C: 45-55.

9 Gottesman O, Scott SA, Ellis SB et al: The CLIPMERGE $P G x$ Program: clinical implementation of personalized medicine through electronic health records and genomics-pharmacogenomics. Clin Pharmacol Ther 2013; 94: 214-217.

10 Johnson JA, Elsey AR, Clare-Salzler MJ, Nessl D, Conlon $M$, Nelson DR: Institutional profile: University of Florida and Shands Hospital Personalized Medicine Program: clinical implementation of pharmacogenetics. Pharmacogenomics 2013; 14: 723-726.

11 Johnson JA, Burkley BM, Langaee TY, Clare-Salzler MJ, Klein TE, Altman RB: Implementing personalized medicine: development of a cost-effective customized pharmacogenetics genotyping array. Clin Pharmacol Ther 2012; 92: 437-439.

12 Pulley JM, Denny JC, Peterson JF et al: Operational implementation of prospective genotyping for personalized medicine: the design of the Vanderbilt PREDICT project. Clin Pharmacol Ther 2012; 92: 87-95.

13 Swen JJ, Wilting I, de Goede AL et al: Pharmacogenetics: from bench to byte. Clin Pharmacol Ther 2008; 83: 781-787.

14 Swen JJ, Nijenhuis M, de Boer A et al: Pharmacogenetics: from bench to byte-an update of guidelines. Clin Pharmacol Ther 2011; 89: 662-673.

15 Altman RB: Pharmacogenomics: "noninferiority" is sufficient for initial implementation. Clin Pharmacol Ther 2011; 89: 348-350.
16 Pirmohamed M, Hughes DA: Pharmacogenetic tests: the need for a level playing field. Nat Rev Drug Discov 2013; 12: 3-4.

17 Pirmohamed M, Burnside G, Eriksson $\mathrm{N}$ et al: A randomized trial of genotype-guided dosing of warfarin. N Engl J Med 2013; 369: 2294-2303.

18 Verhoef TI, Ragia G, de Boer A et al: A randomized trial of genotype-guided dosing of acenocoumarol and phenprocoumon. N Engl J Med 2013; 369: 2304-2312.

19 Coenen MJ, de Jong DJ, van Marrewijk CJ et al: Identification of patients with variants in TPMT and dose reduction reduces hematologic events during thiopurine treatment of inflammatory bowel disease. Gastroenterology 2015; 149: 907-917.e7.

20 Mallal S, Phillips E, Carosi G et al: HLA-B*5701 screening for hypersensitivity to abacavir. N Eng/ J Med 2008; 358: 568-579.

21 Mizzi C, Peters B, Mitropoulou C et al: Personalized pharmacogenomics profiling using whole-genome sequencing. Pharmacogenomics 2014; 15: 1223-1234.

22 Mai Y, Mitropoulou C, Papadopoulou XE et al: Critical appraisal of the views of healthcare professionals with respect to pharmacogenomics and personalized medicine in Greece. Personal Med 2014; 11: 15-26.

23 Mitropoulou C, Mai Y, van Schaik RH, Vozikis A, Patrinos GP: Stakeholder analysis in pharmacogenomics and genomic medicine in Greece. Public Health Genomics 2014; 17: 280-286.

24 Bank PCD, Swen JJ, Guchelaar HJ. An evaluation of Dutch pharmacists' knowledge, experience and attitudes towards pharmacogenetic testing: results of a nation wide survey. Clin Pharmacol Ther 99(Suppl 1): Abstracts: ASCPT 2016(abstract PII-056).

25 Dunnenberger HM, Crews KR, Hoffman JM et al: Preemptive clinical pharmacogenetics implementation: current programs in five US medical centers. Annu Rev Pharmacol Toxicol 2015; 55: 89-106. 